Annales Geophysicae, 23, 3149-3161, 2005

SRef-ID: 1432-0576/ag/2005-23-3149

(C) European Geosciences Union 2005

\title{
Solar weather monitoring
}

\author{
J.-F. Hochedez, A. Zhukov, E. Robbrecht, R. Van der Linden, D. Berghmans, P. Vanlommel, A. Theissen, and F. Clette \\ Royal Observatory of Belgium, Circular Avenue 3, B-1180 Brussels, Belgium
}

Received: 28 February 2005 - Revised: 18 July 2005 - Accepted: 12 August 2005 - Published: 22 November 2005

Part of Special Issue "1st European Space Weather Week (ESWW)"

\begin{abstract}
Space Weather nowcasting and forecasting require solar observations because geoeffective disturbances can arise from three types of solar phenomena: coronal mass ejections (CMEs), flares and coronal holes. For each, we discuss their definition and review their precursors in terms of remote sensing and in-situ observations. The objectives of Space Weather require some specific instrumental features, which we list using the experience gained from the daily operations of the Solar Influences Data analysis Centre (SIDC) at the Royal Observatory of Belgium. Nowcasting requires real-time monitoring to assess quickly and reliably the severity of any potentially geoeffective solar event. Both research and forecasting could incorporate more observations in order to feed case studies and data assimilation respectively. $\mathrm{Nu}$ merical models will result in better predictions of geomagnetic storms and solar energetic particle (SEP) events. We review the data types available to monitor solar activity and interplanetary conditions. They come from space missions and ground observatories and range from sequences of dopplergrams, magnetograms, white-light, chromospheric, coronal, coronagraphic and radio images, to irradiance and in-situ time-series. Their role is summarized together with indications about current and future solar monitoring instruments.
\end{abstract}

Keywords. Solar physics, astrophysics and astronomy (Flares and mass ejections; Energetic particles; Instruments and techniques)

\section{Introduction}

The hostile manifestations of Space Weather can be broadly categorized into geomagnetic storms, large variations of the $\mathrm{X}$-ray to ultraviolet flux (flares) and solar energetic particle events (SEPs).

Correspondence to: J.-F. Hochedez

(hochedez@sidc.be)
Geomagnetic storms are identified using indices like $K_{p}$ or $D_{s t}$. They occur when a sufficiently strong negative (i.e. southward) $B_{z}$ - the North-South component of the interplanetary magnetic field (IMF) - interacts with the Earth's magnetosphere and, to a lesser extent, when the dynamic pressure of the solar wind is enhanced. The main solar phenomena responsible for the appearance of such conditions in the solar wind are Coronal Mass Ejections (CMEs) and high speed flows from Coronal Holes (CHs). CMEs are the main cause of major geomagnetic storms (Gosling et al., 1990; Kahler, 1992; Brueckner et al., 1998; Webb et al., 2000; St. Cyr et al., 2000). Richardson et al. (2001) show that the most intense storms (defined by $K_{p}$ ) at both solar minimum and solar maximum are almost all associated with CMEs.

Flares affect the ionosphere immediately, with adverse effects upon HF communications and radio navigation (GPS and LORAN). Major SEP events are associated with fast CMEs, which are also typically accompanied by flares. Particle acceleration by CME-driven shocks and flares has been proposed, but the relative role of each mechanism, as well as the source of the accelerated particles is a subject of current debate (e.g. Reames, 1999; Kahler et al., 2001; Cane et al., 1988, 2003; Tylka et al., 2002, 2005).

Space Weather entails various solar phenomena and their manifestations in the solar wind to be monitored. Solar monitoring serves both nowcasting and forecasting. Nowcasting operations involve real-time information. To satisfy these needs, monitoring observations must allow the identification of at least one phase of any geoeffective event and the assessment of the severity of those perturbed conditions.

Observations supporting forecasting have to be even more insightful. Instead of the events themselves, forecasters must look for their precursor $(s)$ in a timely manner in order to be able to reliably predict the upcoming event. In addition to operational services, monitoring must in parallel support post-event analysis by supplying germane datasets for case studies. The requisite of forecasting invigorates solar and heliospheric research since more data have to be gathered to hone better predictions. Solar forecasts should ideally be 
quantitative and probabilistic. The modern trend is to assign indices of confidence to the expected solar and geomagnetic activity, events and conditions. Forecasters aim to eventually determine the probability distributions of parameters such as, for CMEs, the time of arrival, the North-South component of the IMF, the dynamic pressure, etc.

Forecasting interplanetary conditions implies nowcasting the Corona. The causality chain actually originates below the photospheric surface, although exploiting local helioseismology is considered speculative at present. Forecasters usually restrict themselves to coronal and photospheric drivers.

The goal of post-event analysis is to assemble a precise global picture of the scenarios that can alter the heliosphere. Modellers aim at developing numerical simulations in order to improve the understanding and the predictions of the solar wind and heliosphere. In this framework, monitoring should lead to data that can be assimilated in numerical codes (Fry et al., 2001; Schrijver and DeRosa, 2003; Luhmann et al., 2004). Monitoring data must also be accumulated and summarized in the long-term to improve our understanding of proxies for e.g. paleo-climatology.

The scope of this paper was originally intended to be the solar sources of Space Weather, but we have broadened this to include other frequently-used monitoring capacities such as in-situ surveillance at $1 \mathrm{AU}$. Nevertheless, the focus remains Solar Weather. In the next three sections, we discuss the main categories of solar phenomena that are relevant to Space Weather, namely: CMEs, flares and CHs. For each, remote sensing and in-situ observations are summarized. Since nowcasting requires recognizing geoeffective circumstances in the midst of otherwise complex and noisy observations, the definition of these circumstances is critical if one is to assess the value of a particular monitoring technique. This point has emerged during the recent years when comparing human and automatic event identifications. In each section, we examine the precursors that allow to forecast the timing and the magnitude of the related phenomenon. The occurrence of geoeffective particle events is usually associated with large CMEs or flares. SEPs will thus be mentioned in the context of their solar origin and not granted a dedicated section. We review the instruments needed to monitor Solar Weather in Sect. 5.

\section{Coronal mass ejections (CMEs)}

\subsection{CME definition and nowcasting}

Flares are not the main cause of interplanetary disturbances producing non-recurrent geomagnetic storms: c.f. "The Solar Flare Myth" (Gosling, 1993, and subsequent debates). The central role is now attributed to events presently known as "Coronal Mass Ejections" (Gosling et al., 1974). CMEs are eruptions of matter from the Sun, typically observed by white-light coronagraphs. Their noteworthy relationship with flares is not well understood and is clearly not one-toone (Kahler, 1992).
Following Hundhausen et al. (1984) and Schwenn (1995), a coronal mass ejection is a new, discrete, bright feature with a radially outward velocity in a white-light coronagraph field of view. Robbrecht and Berghmans (2004) discuss the validity and the limitations of this definition, showing especially that gusty outflows and other LASCO events can conform to it, while not being true CMEs. The orthodox white-light picture of a CME displays a bright leading edge followed by a dark cavity and a central dense core representing an erupted prominence (Crifo et al., 1983; Hundhausen et al., 1987). However, CME observations by LASCO often do not show these three parts clearly. This is especially the case for halo CMEs, when the matter is seen erupting around the entire solar limb: these CMEs travel along the line-of-sight, towards or away from the Earth. They appear faintly in white light because of the angular properties of Thompson scattering and are therefore hard to detect. It is also difficult to make reliable measurements of their physical properties (speed, density, mass, magnetic field...).

CME-related signatures are present in a variety of data, such as radio bursts, which are classified following Wild et al. (1963). Type II radio bursts start at $\sim 100 \mathrm{MHz}$ and then decline in frequency to a few $\mathrm{MHz}$ within typically $30 \mathrm{~min}$. Such radio bursts have been attributed to shocks generated by CMEs which accelerate particles that in turn emit radio waves. As recalled by Jacobs et al. (2005), the frequency drift of the resulting type II radio emissions is related to the dynamics of the shock and the related CME, as the density of the ambient solar wind drops with increasing distance from the Sun. However, the relationship between metric to kilometric type II bursts and CME-produced shocks is not strict (Claßen and Aurass, 2002; Wen and Wang, 2004). Type II events are often accompanied by a parallel burst at the first harmonic (approximately double the frequency) of the main burst. Two or more CMEs colliding in the interplanetary space can cause unusual radio signatures (Gopalswamy et al., 2001b).

In the low Corona, CME onset signatures include filament eruptions, coronal dimmings, EIT waves and post-eruption arcades observed in the EUV and sigmoid-to-arcade restructuring in soft X-rays (Hudson and Cliver, 2001). A CME is often accompanied by several of these signatures but rarely by all together. CMEs are also correlated with fast phenomena visible in $\mathrm{H} \alpha$ such as Moreton waves and filament sudden disappearances (Mouradian et al., 1995). On-disk signatures in $\mathrm{H} \alpha$, EUV or X-rays do not guarantee that a CME has occurred, but they provide a means of identifying those full and partial halo CMEs that are directed toward the Earth (Zhukov et al., 2003; Zhang et al., 2003; Zhukov, 2005). They also help in determining parameters like magnetic field orientation and time of eruption.

In-situ data have a very different character than the information revealed by remote sensing. They are mostly time series that measure the properties of the CME only when it arrives in the vicinity of the Earth. Observations at the L1 Lagrange point, $\sim 1.5$ million kilometres upstream of the Earth, are particularly useful in providing solar wind observations 
$\sim 1 \mathrm{~h}$ before the plasma reaches the Earth (See Weimer et al., 2002, for an exploitation of the time delay between different orbits). The in-situ CME counterparts are termed "interplanetary CME" (ICME). They are characterized by a number of signatures (e.g. Gosling, 1990, and references therein). These may include regions of enhanced (rather stable) magnetic field, decreased proton temperature and high alphaparticle over proton ratio. Counter streaming suprathermal solar wind electrons might be present. Often but not always, the IMF may smoothly rotate through a large angle and the plasma beta is low. These are the characteristics of a magnetic cloud (e.g. Klein and Burlaga, 1982; Burlaga, 2002). Fast ICMEs are usually preceded by a forward shock wave. Between the shock and the magnetic cloud, there is a sheath region where the magnetic field is typically enhanced and may vary rapidly.

The nature of CMEs can be better refined if several complementary observations are examined (Emslie et al., 2004). To predict quantitatively the geospace impact, it is indispensable to gather all possible pieces of information including a knowledge of the magnetosphere into which they propagate. Robbrecht and Berghmans (2005) review the current status of the automated nowcasting capabilities of CMEs.

\subsection{Predicting ICMEs}

Today, the trend is toward more quantitative predictions of, e.g. the time of arrival, the mass, the speed and the $B_{z}$ associated with ICMEs and upstream sheaths. Our current ability to forecast if and when a CME will impact the Earth, as well as its geoeffectiveness, is still relatively poor. Ideally, the Space Weather community needs near-real-time alerts for (partial) halo CMEs that allow sufficient time to run 3-D MHD simulations and thereby estimate their geoeffectiveness.

When a CME is seen in LASCO and determined to be travelling towards the Earth, its speed (projected onto the plane of the sky) and conceivably its acceleration, might be extrapolated to estimate the time of arrival at Earth. The first difficulty here is that the Earthward velocity of a halo CME is difficult to know because it is propagating well out of the plane of the sky. Schwenn et al. (2001) have estimated the line-of-sight CME velocity based on its measured expansion speed. Various models have been proposed to simulate the propagation of the CMEs through the heliosphere, see e.g. Luhmann et al. (2004) and the review by Dryer (2002). The comparison of models with case studies is hampered by the fact that the location of the shock wave relative to the CME is presently unknown and that the relationship between the classical three-part CME structure and the features found in in-situ solar wind measurements is poorly understood. Empirical relations linking the initial velocity of the CME with the time of its travel to the Earth are also of great value. An early example is the " 80 hour rule" (Brueckner et al., 1998) which they suggested "allows one to estimate an early time of a geomagnetic storm's onset (regardless of its initial velocity)" while noting that "this rule will not apply during increasing solar activity, when very fast CMEs are occurring".
And indeed, some extraordinarily fast CMEs reach the Earth in less than $20 \mathrm{~h}$. By contrast, the solar wind transit time from the Sun to the Earth is about $120 \mathrm{~h}$ for a typical velocity of $400 \mathrm{~km} / \mathrm{s}$. Gopalswamy et al. (2001a); Schwenn et al. (2001); Wang et al. (2002); Cho et al. (2003) have derived more complicated relationships between the travel time and the initial CME velocity. Regarding location of the source, Wang et al. (2002) and Zhang et al. (2003) found that CMEs responsible for major geomagnetic storms come mostly from the western hemisphere and within a latitude strip of $\pm 30^{\circ}$ around the equator.

The strength of the southward magnetic field associated with ICMEs is an important parameter for predicting geoeffectiveness. Lindsay et al. (1999) found a relationship between CME speed and magnitude of the total magnetic field related to the ICME and therefore also with the intensity of geomagnetic storms. A method of predicting $B_{z}$ directly remains elusive. The orientation of the neutral line of the CME source region (when bipolar) in the photospheric magnetograms can provide one indicator of the ICME field direction. Bothmer and Rust (1997) pointed out that the direction of the internal magnetic field of the ICME associated with the January 1997 halo CME could be inferred from the solar magnetic field structure at the site of the associated filament. A one-to-one correspondence between filament chirality (Chae, 2000; Pevtsov et al., 2003) and the sign of magnetic helicity in interplanetary CMEs has been reported in a number of studies (Bothmer and Schwenn, 1994; Marubashi, 2000; Yurchyshyn et al., 2001; McAllister et al., 2001). These findings represent a great step forward for Space Weather predictions since they show that magnetic fields within ICMEs may be predictable from measurements of the solar magnetic field and simultaneous coronal observations. Jing et al. (2004) illustrate the prediction of geoeffective events on the basis of the chirality of the associated filament and its magnetic field orientation. However, strong magnetic fields, and in particular strong negative $B_{z}$, can stem not only from the plasma ejected from the Sun, but also from compression of the ambient solar wind by CMEdriven shocks

\subsection{Predicting CMEs}

We have discussed above the prediction of ICMEs at Earth based on prior CME observations. A fundamental advance would be the ability to forecast CMEs before they are seen in a white-light coronagraph. Until recently, this has not been really implemented by Space Weather forecasters, but this could change (Jing et al., 2004). The operational motivation might appear moderate given the significant time available before a LASCO CME reaches the Earth (typically 2-3 days). However, ideal forecasting would predict fast CMEs as well as flares because both may generate dangerous quasiimmediate proton storms (Cliver et al., 2004).

CMEs are often closely linked with prominence eruptions and hence their prediction requires identifying the symptoms which suggest that filaments seen in chromospheric or 
coronal images are about to become unstable. Many different CME initiation scenarios have been suggested and it is a challenge to validate or falsify them (Sterling and Moore, 2004). Theoretical models have been reviewed by Klimchuk (2000). Based on basic physical properties, such as energetics, structure and dynamics, he distinguished five models which can be divided into two types 1) directly driven models and 2) storage and release models. However, a comprehensive statistical study on CME speeds has provided no evidence for the existence of these two classes (Yurchyshyn et al., 2005).

The use of sigmoids has also been suggested for CME predictions. Sigmoids are S- or reverse S-shaped regions (therefore with highly non-potential magnetic configurations) observed by soft X-ray telescopes. It has been suggested (Canfield et al., 1999) that sigmoidal active regions are more likely to erupt, provided that the S-shape is not a mere projection effect (Glover et al., 2000). Some doubts have appeared about this proposal (Zhukov, 2005). Another track for CME predictions uses the magnetic helicity of coronal structures estimated from photospheric magnetograms. When the helicity exceeds a certain threshold, the kink instability makes the whole structure erupt (Rust, 2002). Other approaches have been based upon vector magnetograms (Falconer, 2001). Numerical methods incorporating the photospheric vector magnetic field offer high prospects of success (Abbett et al., 2004). In addition, several more unusual possible precursors have been noted, for example Kaufmann et al. (2003) suggest that rapid solar spikes at submillimeter waves would offer an early signature of CMEs.

\section{Flares and spectral irradiance variability}

\subsection{Flare definition and nowcasting}

In the present paper, flares are conceived as purely radiative events and they are thus associated to irradiance variations. They are defined by "large" and "sudden" increases of the photon flux. These bursts are best observed in X-ray and ultraviolet, but also in $\mathrm{H} \alpha$ or even exceptionally in whitelight, by radiometers or imaging telescopes. However, at some level, it is arbitrary to discriminate a small flare against the background baseline (Veronig et al., 2004). Just as with CMEs, it becomes necessary to assemble various observations to decide upon the physical nature of the irradiance variation. Big flares are so obvious that their definition is not an issue. However, the detection of small flares has deep fundamental implications (Buchlin et al., 2005) and could contribute to CME and larger flare forecasting (Hochedez et al., 2002). Regarding the in-situ counterparts of flares, there are none if we stick to the radiative definition. It is nevertheless customary to mention the flare-produced impulsive SEPs arriving with a typical delay of minutes to few hours after the flare (Krucker and Lin, 2000).

Type III radio bursts are associated with the impulsive and early phase of flares. They start at a few $100 \mathrm{MHz}$ and then rapidly decline in frequency to a few $\mathrm{MHz}$ within typically $5 \mathrm{~min}$. They are caused by near-relativistic electrons moving along open magnetic field lines from flaring centres close to the Sun into lower density regions. The acceleration of the electrons is a result of magnetic reconnection, which also causes a downward stream of electrons into lower and denser regions of the atmosphere, giving rise to $\mathrm{X}$-ray emission (Bastian et al., 1998). Cane et al. (2002) show radio spectra of flares together with their X-ray light curves, evidencing a new class of type III-like emissions (termed type-III-1) that accompanies essentially all major SEP events. It proves the existence of open field lines along which flare electrons and possibly ions escape to the interplanetary medium. Flare particles can hence contribute to major SEP events.

\subsection{Predicting flares}

The incentive to predict flares is high due to the immediacy and the threat of the potential effects of flares on spacecrafts, astronauts, aircraft passengers, etc. (Feynman and Gabriel, 2000). Flare warning services currently use sunspot group classifications such as McIntosh's or Mount Wilson's (Hale et al., 1919; McIntosh, 1990) or use recent flaring history to deliver an empirical global risk index (Wheatland, 2004; Gallagher et al., 2002). Enhanced mass motion in an active region observed in e.g. H $\alpha$ can also be a useful pre-flare signature (Gaizauskas, 1989). Several different types of data, typically from seismology (Haber et al., 2003), magnetographs (Sammis et al., 2000) and coronal imagers would have to be assimilated by models to bring about significant progress on flaring prediction. Complementary promising tracks include data mining techniques (Núñez et al., 2005). Long-term prediction of the spectral irradiance is outside the scope of this paper.

It is also difficult to predict SEP spectra and fluxes. The charged particles propagate along the interplanetary magnetic field lines, which have the average form of an Archimedian (Parker) spiral. Flares situated between the central meridian and the western limb are therefore more likely to have a prompt impact on the radiation environment near the Earth (Cane et al., 1988; Ippolito et al., 2005). The penetration of energetic particles into the Earth's magnetosphere is a separate problem, not discussed here.

\section{Coronal holes (CHs) and co-rotating interaction regions (CIRs)}

\subsection{CH and CIR definition and nowcasting}

A coronal hole continuously emits fast solar wind, which interacts with the slow wind forming a Co-rotating Interaction Region (CIR) at the stream leading edge. CIRs typically only fully develop beyond Earth's orbit. The signatures of the evolving CIRs and flows from coronal holes were described in the 1970s when the Pioneer 10/11 and Voyager spacecrafts explored the heliosphere (Belcher et al., 1969; Tsurutani et al., 1995). First, the region of enhanced density 
and magnetic field arrives while the solar wind velocity remains rather low. These enhancements are formed because of compression of the slow wind plasma and magnetic field by the upcoming fast flow. The sign of the IMF $\mathrm{B}_{x}$ component can change around this time (sector boundary). Then, the velocity rises as the flow from the coronal hole arrives. In addition to increased dynamic pressure, this carries strong Alfvén waves with oscillating $B_{z}$. Although the periods of sufficiently strong southward IMF are very short $(\sim 1 \mathrm{~h})$, the multiple oscillations together may result in appreciable and long-lasting geomagnetic disturbances, including high values of the auroral electrojet $(A E)$ index.

Coronal holes are seen in solar X-ray or EUV images as extended darker volumes because they contain cooler, less dense plasma. But these observations do not prove directly that they are the only regions of open field lines. Narrow coronal holes can be reliably discriminated from filaments (which are also dark in EUV) using observations in $\mathrm{H} \alpha$, He I $(1083 \mathrm{~nm})$ and in photospheric magnetograms.

\subsection{Predicting the geoeffectiveness of coronal holes $(\mathrm{CH})$}

Predicting intervals of geoeffectiveness for $\mathrm{CHs}$ might be expected to be relatively easy because of their slow evolution, dominated by the global solar rotation. The 27-day recurrence helps in predicting $\mathrm{CH}$ effects. Furthermore, $\mathrm{CH}$ can be monitored for several days before they become most geoeffective after crossing the central meridian. The Sun-Earth magnetic connectivity is unfortunately not straightforward (e.g. Schwadron and McComas, 2004). Forecasters have to assume that magnetic fields and flows follow an average Parker spiral, while this is only an approximation. Progress is currently needed on three issues. These are the mechanisms driving the evolution of coronal hole 3-D geometry (including the possible vicinity of ARs), the knowledge of the instantaneous Parker spiral geometry and the real-time measurement of the fast wind speed before it is sensed near the Earth.

\section{Monitoring capabilities}

\subsection{Observational features needed for solar monitoring}

Recapitulating the previous three sections, the observations that are both practicable and necessary for Solar Weather nowcasting and forecasting are photospheric dopplergrams for helioseismology, photospheric magnetograms, photospheric White Light images, chromospheric images, cool Corona images, hot Corona images, disc integrated irradiance, spectrally resolved or not, upper Corona "coronagraphic" images, radio measurements and in-situ measurements. Before visiting every type of data in terms of its primary role, present and future availability, we first detail the generic features that are specially required by Solar Weather monitoring. This builds upon a table by Daly (2002), but is further specified here.
1. A key feature of monitoring is the continuity of observations. Ground observatories have therefore to form international networks delivering homogeneous records in order to cope with the day-night cycle and adverse weather conditions at individual observatories (e.g. Steinegger et al., 2001). As far as space missions are concerned, the continuity requirement translates into more costly orbits (cf. http://sdo.gsfc.nasa.gov/sdo_ mission_specs.htm \#ORBIT or Domingo et al. (1995)) and using instruments of similar heritage. Though spacecraft payloads do vary, we anticipate that standards will emerge that will provide consistency in nowcasting and forecasting performance and objectives (e.g. Lemen et al. (2004) or Defise et al. (2004)). Researchoriented Space Weather experiments will naturally depart from such standards.

2. Regarding data availability, we distinguish between the needs for operative monitoring and for post-event analysis. For the former, the observations must be accessible to users in near real-time (Kunches, 2002), even from a deep space probe such as STEREO (Biesecker and Webb, 2004). Consistent standards must be applied when reformatting the raw data into exploitable higher level products using physical units.

3. Finally, the spatial and temporal resolution of the observations must become progressively finer as the objective goes from nowcasting, to forecasting and then research. Solar Weather is mostly about dynamical disturbances and hence about temporal evolution. The observational cadence has to be higher for research and forecasting than for nowcasting.

In the rest of this section, we discuss the main instrumental techniques employed in monitoring solar and interplanetary conditions. The review will highlight the primary role of each type of data and some available instruments. Complementary information is linked through http: //sidc.be/, "Related links", or http://solar.physics.montana. edu/max_millennium/obs/.

\subsection{Helioseismology}

Dopplergrams capable of monitoring helioseismology have been available since January 1996 from the Michelson Doppler Imager (MDI) (Scherrer et al., 1995) and since October 2001 from the GONG+ network (GONG+ Team (1999); Hill et al. (2003) and http://gong.nso.edu/). In the future, the HMI (Scherrer and SDO/HMI Team (2002); http://hmi.stanford.edu/) on board SDO (the Solar and Dynamics Observatory, to be launched in April 2008) will bring the prospect of far surface imaging and Solar Subsurface Weather (Toomre, 2003).

Following Braun and Lindsey (2001) and references therein, helioseismic holography has developed as a general diagnostic tool for local helioseismology, with the purpose of imaging acoustic sources in the solar interior and on the 
far surface of the Sun. Holographic procedures may be employed to produce diffraction-limited images of the entire far surface (and poles) of the Sun. To facilitate Space Weather forecasting, seismic images of the central portion of the solar far side have been produced nearly continuously since late 2000 by a synoptic program that applies phase sensitive holography to "quicklook" medium-resolution data from MDI (http://soi.stanford.edu/data/farside/).

According to Braun and Lindsey (2000), Haber et al. (2003) and Dzifcáková et al. (2003), local Helioseismology has the potential to provide flow and sound-speed maps of the upper convection zone in near-real time for Space Weather applications. It is believed that subphotospheric shearing flows play an important role in creating unstable magnetic topology that leads to the initiation of flares and CMEs. However, the relationship between the flows and flaring activity is not yet well understood.

\subsection{Photospheric magnetograms}

As they are key to inferring foot-point motions, evaluating active region complexity, permitting MHD extrapolations, assessing helicity and so on, line-of-sight magnetograms are vital for Solar Weather. The related literature is very abundant, examples being Feynman and Martin (1995) and Wang et al. (1996).

Regular observations of high-resolution full-disk magnetograms are extremely valuable. Thanks to the SOHO orbit around the L1 Lagrange point, quasi-uninterrupted full-Sun magnetograms come from MDI (Scherrer et al., 1995) with a $4^{\prime \prime}$ resolution and a 1 min cadence. Nevertheless, groundbased magnetographs are used too. The Kitt Peak Vacuum Tower (KPVT) of the National Solar Observatory (NSO) has provided magnetograms since 1974 . The quality improved when the Spectromagnetograph (SPM) became operative (Jones et al., 1992) producing a $2.28^{\prime \prime}$ resolution image every $40 \mathrm{~min}$. The GONG+ network (Big Bear, Cerro Tololo, and Learmonth) provides one magnetogram every minute continuously with a spatial resolution of the order of $5^{\prime \prime}$ since October 2001. GONG+ and SPM observations have been shown to agree, but they underestimate fluxes by $20-40 \%$ as compared to MDI (Thornton and Jones, 2002). Magnetograms at lower resolution are regularly recorded at the Wilcox Solar Observatory and at the Mount Wilson Observatory. ISOON (http://www.nso.edu/nsosp/isoon/description. $\mathrm{html}$ ) is a semi-autonomous, remotely-controlled patrol set of telescopes, currently limited to a single site, the National Solar Observatory at Sacramento Peak. ISOON acquires solar images in the $\mathrm{H} \alpha$ line (once per minute), in continuum (once every ten minutes), and in line-of-sight magnetic fields. Additional images or alternate observing sequences are programmable options. The ground-based SOLIS facility (http://solis.nso.edu/) produces highly valuable data, including vector magnetograms. The future HMI on SDO will also be a vector magnetograph. Its instrumental features can be found at: http://hmi.stanford.edu/. HMI will help to reduce the current limitations in the routine availability of vector magnetograms and in the accuracy of their transverse field measurements.

\subsection{Photospheric white-light images}

MDI also currently provides continuous white-light images from space and HMI will take over this role when SDO is launched. However, many ground telescopes around the world observe the Sun, such as the ones operated by the SIDC (Berghmans et al., 2005a, and http://sidc.oma.be). White light solar disc monitoring is useful for nowcasting the infrequent white light flares (WLF) and for classifying active regions. Large sunspots have been recognized to flare more (Canfield et al., 1999) offering a cheap and dependable method of forecasting. Nevertheless, cadences higher than those achieved routinely might give further insight into e.g. the evolution of delta spots, peculiar photospheric velocity fields (Zuccarello, 1992) and subsequent activity.

\subsection{Chromospheric images}

Chromospheric image sequences are especially effective for monitoring filaments by detecting their presence, their sudden disappearance and estimating their chirality (Pevtsov et al., 2003) through $H \alpha$ observations. Other transients can be observed, such as Moreton waves.

The global high-resolution $\mathrm{H} \alpha(656.3 \mathrm{~nm})$ network (http: //www.bbso.njit.edu/Research/Halpha/ and Steinegger et al., 2001)) utilizes facilities at the Big Bear Solar Observatory (BBSO) in California, the Kanzelhöhe Solar Observatory (KSO) in Austria, the Catania Astrophysical Observatory (CAO) in Italy, the Meudon Observatory in France, the Huairou Solar Observing Station (HSOS) and the Yunnan Astronomical Observatory (YNAO) in China. These observatories have around 300 sunny days a year, good seeing conditions, adequate observing staffs and well established $\mathrm{H} \alpha$ telescope systems. Each of the three stations has a $1 \mathrm{k}^{2}$ or $2 \mathrm{k}^{2} \mathrm{CCD}$ detector to monitor the Sun with a spatial resolution of 2 arc s. Observations of 1 min cadence are obtained at each station. Higher cadences can be triggered by automated filament eruption detection. In summer, each station can observe $12 \mathrm{~h}$ on clear days and there should ideally be no gap between observations at adjacent stations. In winter, when each station is expected to operate $8 \mathrm{~h}$, the BBSO/YNAO gap is about $1.6 \mathrm{~h}$ and the BBSO/KSO gap about $0.7 \mathrm{~h}$. Based on weather records at the three stations, the duty cycle is approximately $70 \%$ in summer and $60 \%$ in winter.

$L y \alpha$ imaging would also be an interesting diagnostics tool, but it necessitates a space telescope. Other wavelengths, such as Ca II K at e.g. BBSO, or He I (1083 nm) at e.g. Kitt Peak can be of interest for monitoring the chromosphere. He I is particularly useful to distinguish between filaments and coronal holes or filament channels, which all appear dark in coronal EUV images. 


\subsection{Cool Corona images}

The strong need for imaging the cool Corona has been stated in previous sections of this paper. Given that the wavelength range is in the extreme ultraviolet, this type of observation has to be made from space. Today, the EIT (Delaboudiniere et al., 1995) onboard SOHO provides synoptic $1024^{2}$ images every $6 \mathrm{~h}$ in three coronal passbands corresponding to temperatures ranging from 1 to $2 \mathrm{MK}$ and in the He II-dominated channel, which corresponds to the lower Transition Region regime. In addition, a "CME watch" mode runs continuously, offering a cadence better than $20 \mathrm{~min}$ in Fe XII at $19.5 \mathrm{~nm}$, one of the 3 coronal channels. The resulting monitoring data play a crucial role in solar nowcasting and forecasting. During the intervals (the "keyholes") when the SOHO mission cannot downlink observations, the SIDC (Clette et al., 2002; Berghmans et al., 2005a) makes use of SPIRIT-CORONAS F movies (Zhitnik et al., 2002) thanks to a special agreement with the Russian PI institute.

There is currently no other full Sun EUV imager, and we need to rely on EIT and SPIRIT until EUVI of SECCHISTEREO, SWAP aboard the ESA PROBA 2 and AIA-SDO enter operations. EUVI, the Extreme Ultraviolet Imager (Wuelser et al., 2004), is part of the SECCHI suite of the NASA STEREO mission. Identical telescopes on the two STEREO spacecraft will study the solar Corona in three dimensions, and specifically focus on the initiation of CMEs. The EUVI $2048^{2}$ detectors have a field-of-view out to 1.7 solar radii, and observe in four spectral channels that span the 0.1 to $20 \mathrm{MK}$ temperature range. In addition to its view from two vantage points, the EUVI will provide a substantial improvement in image resolution and image cadence over SOHO-EIT. In 2007, SWAP (Berghmans et al., 2005b) will bring advances thanks to its 1 min cadence, autonomous CME-tracking ability and concomitancy with EUVI. Its passband is centred on $17.5 \mathrm{~nm}$. SWAP will demonstrate the Solar Weather potential of a micro-mission owing to its original optical design, CMOS-APS detectors and onboard image processing capabilities. The impressive AIA (http://aia. lmsal.com/) will snapshot the solar atmosphere with extreme spatial $\left(4 \mathrm{k}^{2}\right)$ and temporal $(10 \mathrm{~s})$ resolutions. It will also sample 8 temperature ranges for $\log \mathrm{T}$ in the interval (3.7-7.2). When it operates, AIA will become central in research as well as solar weather applications.

\subsection{Hot Corona images}

Hot coronal movies provide an exclusive view on coronal holes, sigmoids, cusps, flares, post-eruptive arcades, etc. The Soft X-ray Telescope (SXT) on board Yohkoh (Tsuneta et al., 1991), and today the SXI series (Lemen et al., 2004; Nitta et al., 2004) on the GOES $12, \mathrm{~N}$ and $\mathrm{O}$ are monitoring the multi-million degree Corona. The Yohkoh satellite completed its mission on 14 December 2001. SXT observed the dynamic solar Corona nearly continuously for more than 10 years, leading to many important discoveries. Since September 2001, soft X-ray images of the Sun are taken by SXI at a 1-min cadence with a $512^{2}$ intensified CCD. The X-ray telescope (XRT) of Solar-B (to be launched in late 2006) will provide full disk, soft X-ray images with twice the spatial resolution of the Yohkoh SXT and enhanced sensitivity to wavelengths above $4 \mathrm{~nm}$. Although not a Solar Weather dedicated mission, the Rhessi team updates a list of flare events: http://hesperia.gsfc.nasa.gov/hessidata/ dbase/ and http://hessi_flare_list.txt.

\subsection{Disc integrated irradiance time-series}

Irradiance measurements are of interest in two areas of Space Weather. In the short term, they allow the detection of flares that are large enough to disturb the full-disc integrated signal, which is more easily achieved towards shorter wavelengths (cf. the GOES flare classification). In the long term, they form a vital input to aeronomy models.

The energy of solar radiation reaching the Earth is in most parts of its spectral range absorbed and deposited in various layers of the terrestrial atmosphere. Radiation including the vacuum UV is of special interest for Space Weather and is highly variable. Therefore, various space-based missions monitor the spectral irradiance (See Hochedez et al., 2005, for a summary). Most missions cover a limited optimised spectral range, with the recent TIMED-SEE mission closing a gap in the mostly unmonitored EUV spectral range (Woods et al., 2005; Judge et al., 2001). It should be noted that a high-cadence, high-spectral resolution irradiance monitoring is a challenging task.

Workarounds against such gaps consist of using as proxies, measurements of other spectral features correlated to UV or X-ray radiation. These include solar $10.7 \mathrm{~cm}$ radio flux (F10.7), He 1083 equivalent width and the Mg II core-towing ratio (See Floyd et al., 2005). Solar irradiance models are built using these proxies (see e.g. the Solar2000 model, Tobiska, 2004). Another model employs empirical differential emission measures to induce line intensities of unobserved UV and X-ray lines (the NRLEUV model, Warren et al., 2001). Different solar irradiance models unfortunately differ extensively (up to $40 \%$ ) in their predicted intensities (Lean et al., 2003) for two major reasons. First, the data on which these models are based suffer from calibration problems. Second, no single mission or ensemble of missions has ever measured the entire spectral range long enough to provide a consistent monitoring of the solar irradiance over a time scale corresponding to a solar cycle. In order to tackle the first problem, Proba2-LYRA (Hochedez et al., 2005) and Picard-PREMOS (http://www.pmodwrc.ch/ pmod.php?topic=proj_space_premos), for instance, employ a redundancy strategy to continuously calibrate the detectors and to monitor their degradation with time.

\subsection{Upper Corona (coronagraphic) images}

Coronal mass ejections are routinely observed by the LASCO C2 and C 3 coronagraphs onboard SOHO (Brueckner et al., 1995). The observation of halo CMEs by LASCO, 
and their classification with the help of EIT as either Earthdirected or backside has been a great breakthrough in the forecasting of geomagnetic storms. The LASCO coronagraphs are currently our only means to probe the solar Corona within $\sim 2-30 R_{\odot}$.

In February 2006, the two STEREO spacecraft will be launched with a SECCHI suite on each (Howard et al., 2000), including a set of 2 overlapping coronagraphs and 2 heliospheric imagers (HIs) such that the whole path from the Sun to the Earth will be covered. The HIs are the first instruments of their kind and offer an interesting perspective for ICME forecast since they could provide additional constraints to numerical codes that currently run only with coronal boundary conditions. The two STEREO spacecraft will lead ahead and trail behind the Earth with a mutual separation angle that increases by $45^{\circ}$ per year. This implies however that, while STEREO is a most exciting scientific mission, it will remain difficult to use it as an operative monitoring mission since the perspective changes from day to day and the mission duration is inherently limited.

Eyles et al. (2003) describe a white light instrument (SMEI) specifically designed to detect and forecast the arrival of CMEs and other heliospheric structures moving towards the Earth. SMEI was launched in January 2003 into a Sun-synchronous polar orbit. The instrument scans most of the sky every 102-min orbit. Full sky images are available at this cadence since March 2003: http://smei.nso.edu/. This type of measurement requires a careful design and implementation, but it delivers unique coronagraphic observations, not widely used in real time though.

\subsection{Radio measurements}

Easily accessible from the ground, the metric radio domain of the electromagnetic spectrum is monitored by numerous observatories for signatures of shock waves and fast moving electrons. Such observatories comprise facilities like the US Air Force Radio Solar Telescope Network, Potsdam, Culgoora, Learmonth, and many others. However, to monitor decametric to kilometric radio bursts, one must rely on space missions such as WAVES on Wind (Bougeret et al., 1995).

In contrast to coronagraphic observations, radio observations of CMEs (Gopalswamy and Kundu, 1992) enable the early detection of CMEs, only when they appear on-disk and are directed towards the earth. A study of radio on-disk CMEs has recently been presented by Ramesh et al. (2003). Coronal Holes (Moran et al., 2001) are apparent on radio images as radiation enhancements. Radio imaging facilities used for monitoring include NoRH (the Nobeyama Radioheliograph, Nakajima et al., 1995) and the Siberian Solar Radio Telescope (Grechnev et al., 2003). The next big stride in radio interferometry may be FASR (the Frequency Agile Solar Radiotelescope, http://www.ovsa.njit.edu/fasr/).

As to the $10.7 \mathrm{~cm}$ flux measurements, they were made near Ottawa, Canada from 1947 to 1990 . Since 1990 they are carried out at Penticton, Canada (http://www.drao-ofr.hia-iha. nrc-cnrc.gc.ca/icarus/www/sol_home.shtml).
As an example of the application of using interplanetary scintillation (IPS), the Low Frequency array (LOFAR) will be a next generation digital aperture synthesis radio telescope covering the frequency range from 10 to $240 \mathrm{MHz}$. The instrument is currently in its design phase. Oberoi and Kasper (2004) highlight the solar, heliospheric and Space Weather applications where LOFAR can provide useful information inaccessible by any other means. The techniques of interest include tracking coronal mass ejections out to large distances using IPS methods, tomographic reconstruction of the solar wind in the inner heliosphere using IPS and direct imaging of the radio emission from CMEs.

\subsection{In-situ measurements}

In-situ monitoring of the solar wind plasma and interplanetary magnetic field parameters is very important as it currently provides the input for quantitative models of the solar wind interaction with the Earth's magnetosphere. These models may include the prediction of geomagnetic indices (See e.g. Temerin and $\mathrm{Li}, 2002$ ). The main source of insitu data is currently the Advanced Composition Explorer (ACE) spacecraft (Stone et al., 1998, http://www.sec.noaa. gov/ace/). Since early 1998, the ACE instruments (MAG, SWEPAM, EPAM, SIS, SWICS, SWIMS, ULEIS, etc.) have delivered continuous measurements of solar wind and energetic particle parameters. Being positioned at an orbit around $\mathrm{L} 1$, its real-time data give us about $1 \mathrm{~h}$ warning of solar wind structures approaching the Earth. Besides identifying those features that drive geomagnetic storms, this set of instruments provides the data for quantitative models. SOHO also possesses an in-situ payload package with CELIAS, COSTEP and ERNE. The CELIAS solar wind plasma experiment (Judge et al., 2001) has the advantage that it is more resilient than ACE-SWEPAM to SEP events. Occasionally, at times of high SEP intensities, which are also often critical times for Space Weather forecasting, ACE solar wind plasma data are not routinely available, while CELIAS continues to function well. WIND and GEOTAIL also measure interplanetary plasma and magnetic fields but in-situ monitoring is not available when inside or close to the magnetosphere. From December 2001 to April 2004, GENESIS (http://www.genesismission.org/) provided very good data near L1 before it was returned to Earth. Thus, although ACE is now in good working condition, it is unique and no replacement is currently foreseen.

\subsection{Miscellaneous}

A few instruments do not easily fit the main categories discussed above. First we mention the SWAN instrument on SOHO. Quémerais and Bertaux (2002) present a method to forecast solar indices on time scales of half a solar rotation. Their method uses the Ly $\alpha$ interplanetary glow data obtained by SOHO-SWAN. Active regions are brighter than the Quiet Sun in Ly $\alpha$. Resonance scattering creates an excess of illumination related to active regions. They derive 
a far-side to near-side flux ratio used to predict the evolution of solar indices such as the solar $L y \alpha$ irradiance, the $10.7 \mathrm{~cm}$ radio flux or the $\mathrm{Mg}$ II solar indices. This technique is used to improve the quality of Space Weather forecast (http://sohowww.nascom.nasa.gov/data/summary/swan/).

Ground-based observations of cosmic rays by neutron or muon monitors have also been used for Space Weather monitoring. Jansen Frank et al. (2005) discuss the benefits that an instrument like MUSTANG (the Muon Spaceweather Telescope for Anisotropies at Greifswald) will bring. It measures the anisotropy of galactic cosmic rays that are caused by CMEs and can provide information about their position and geometry. It will hence give advanced warnings of the arrival of plasma clouds at the Earth (See also Leerungnavarat et al., 2003).

\section{Conclusions}

In the above discussion, we have noted a number of limitations of current capabilities. The first one is the imperfect monitoring of Solar Weather events. The performance (sensitivity, resolution, cadence) of most current observations must improve to characterize better the potentially geoeffective circumstances. Furthermore, there exists a gap in the immense region of space between wide field coronagraphs $\left(\sim 30 \mathrm{R}_{\odot}\right)$ and in-situ payloads $\left(\sim 215 \mathrm{R}_{\odot}\right)$. STEREO-HI will fill this gap and we anticipate learning much from its observations. Even with perfect data, forecasts would have a limited predictive horizon because solar and heliospheric physics is not fully deterministic. Therefore, Space Weather forecasting shall always be probabilistic. As a second consequence, nowcasting needs continuous updating to account for the unpredictable events.

Despite the above restrictions, important progress is ongoing. Forecasting is evolving from "real-time data-driven" towards "real-time model-driven". Inputs specific to these numerical models must be gathered. Several models already run in near-real time (Rapid Prototyping Center, http: //www.sec.noaa.gov/rpc/). However, most of them simulate the region between L1 and the Earth and not between the Sun and L1. In this frame, Solar Weather monitoring is the activity, which records all necessary data to model the next state of the Sun and Heliosphere.

Solar Weather monitoring is likely to receive increasing programmatic attention in the future. An ambitious Solar Weather plan could be made rightfully comprehensive, but then costly. Additionally, as-yet-unexpected monitoring requirements may arise. But some good monitoring capabilities can be relatively cheap and redundancy is then affordable. Open data policy and database technologies should naturally prevent excessive redundancies and induce the best possible international solar monitoring network. However, there is no backup sometimes. Despite their truly unique and critical character, this is currently the case for the LASCO coronagraphs or the ACE payload, unless a mission such as the Chinese $\mathrm{KuaFu}$ succeeds in bridging the observations.
Concerning space instruments, a series of moderate missions is perhaps the winning concept. These considerations could be useful when ESA or the European Union define their possible contributions to international Space Weather facilities.

Acknowledgements. Funding of J. F. Hochedez, A. Zhukov, E. Robbrecht, P. Vanlommel, A. Theissen by the Belgian Federal Science Policy Office (BELSPO) through the ESA/PRODEX programme is hereby appreciatively acknowledged. This work takes place in the frame of the Cost 724 action. The authors thank the anonymous referees for their numerous comments and suggestions for improvement.

Topical Editor R. J. Forsyth thanks J. M. Kunches and I. G. Richardson for their help in evaluating this paper.

\section{References}

Abbett, W. P., Mikić, Z., Linker, J. A., McTiernan, J. M., Magara, T., and Fisher, G. H.: The photospheric boundary of Sun-toEarth coupled models, J. Atmos. S.-P., 66, 1257-1270, 2004.

Bastian, T. S., Benz, A. O., and Gary, D. E.: Radio Emission from Solar Flares, Ann. R. Astron. Astr., 36, 131-188, 1998.

Belcher, J. W., Davis, L. J., and Smith, E. J.: Large-Amplitude Alfvén Waves in the Interplanetary Medium: Mariner 5, J. Geophys. Res., 74, 2302, 1969.

Berghmans, D., Van der Linden, R., Vanlommel, P., Warnant, R., Zhukov, A., Robbrecht, E., Clette, F., Podladchikova, O., Nicula, B., Hochedez, J.-F., Wauters, L., and Willems, S.: Solar activity: nowcasting and forecasting at the SIDC, Ann. Geophys., 23, 3115-3128, 2005a.

Berghmans, D., Hochedez, J.-F., Defise, J. M., Lecat, J. H., Nicula,, B., Slemzin, V., Lawrence, G., Katsyiannis, A. C., Van der Linden R., Zhukov, A., Clette, F., Rochus, P., Mazy, E., Thibert, T., Nicolosi, P., Pelizzo, M.-G., and Schühle, U.: SWAP onboard PROBA 2, a new EUV imager for solar monitoring, Adv. Space Res., accepted, 2005b.

Biesecker, D. A. and Webb, D. F.: Space Weather Aspects of the STEREO Mission, AGU Fall Meeting Abstracts, p. B412, 2004.

Bothmer, J. and Rust, V. D.: Coronal Mass Ejections, (Eds.) Crooker, N., Joselyn and J. Feynman, AGU Geophys. Monogr., 99, 139, 1997.

Bothmer, V. and Schwenn, R.: Eruptive prominences as sources of magnetic clouds in the solar wind, Space Sci. Res., 70, 215-220, 1994.

Bougeret, J.-L., Kaiser, M. L., Kellogg, P. J., Manning, R., Goetz, K., Monson, S. J., Monge, N., Friel, L., Meetre, C. A., Perche, C., Sitruk, L., and Hoang, S.: Waves: The Radio and Plasma Wave Investigation on the Wind Spacecraft, Space Sci. Res., 71, 231-263, 1995.

Braun, D. C. and Lindsey, C.: Helioseismic Holography of ActiveRegion Subphotospheres - (Invited Review), Sol. Phys., 192, 285-305, 2000.

Braun, D. C. and Lindsey, C.: Seismic Imaging of the Far Hemisphere of the Sun, Astrophys. J. L., 560, L189-L192, 2001.

Brueckner, G. E., Howard, R. A., Koomen, M. J., Korendyke, C. M., Michels, D. J., Moses, J. D., Socker, D. G., Dere, K. P., Lamy, P. L., Llebaria, A., Bout, M. V., Schwenn, R., Simnett, G. M., Bedford, D. K., and Eyles, C. J.: The Large Angle Spectroscopic Coronagraph (LASCO), Sol. Phys., 162, 357-402, 1995. 
Brueckner, G. E., Delaboudiniere, J.-P., Howard, R. A., Paswaters, S. E., St. Cyr, O. C., Schwenn, R., Lamy, P., Simnett, G. M., Thompson, B., and Wang, D.: Geomagnetic storms caused by coronal mass ejections (CMEs): March 1996 through June 1997, Geophys. Res. Lett., 25, 3019-3022, 1998.

Buchlin, E., Galtier, S., and Velli, M.: Influence of the definition of dissipative events on their statistics, Astron. Astr., 436, 355-362, 2005.

Burlaga, L. F.: Review of Magnetic Clouds/Flux Ropes and Types of Ejecta, Bulletin of the American Astronomical Society, 34, $752,2002$.

Cane, H. V., Reames, D. V., and von Rosenvinge, T. T.: The role of interplanetary shocks in the longitude distribution of solar energetic particles, J. Geophys. Res., 93, 9555-9567, 1988.

Cane, H. V., Erickson, W. C., and Prestage, N. P.: Solar flares, type III radio bursts, coronal mass ejections, and energetic particles, J. Geophys. Res. (Space Phys.), 107, 14-1-14-19, 2002.

Cane, H. V., von Rosenvinge, T. T., Cohen, C. M. S., and Mewaldt, R. A.: Two components in major solar particle events, Geophys. Res. Lett., 30, 5-1-5-4, 2003.

Canfield, R. C., Hudson, H. S., and McKenzie, D. E.: Sigmoidal morphology and eruptive solar activity, Geophys. Res. Lett., 26, 627-630, 1999.

Chae, J.: The Magnetic Helicity Sign of Filament Chirality, Astrophys. J., 540, L115-L118, 2000.

Cho, K.-S., Moon, Y.-J., Dryer, M., Fry, C. D., Park, Y.-D., and Kim, K.-S.: A statistical comparison of interplanetary shock and CME propagation models, J. Geophys. Res., 108, 8-1, 2003.

Claßen, H. T. and Aurass, H.: On the association between type II radio bursts and CMEs, Astron. Astr., 384, 1098-1106, 2002.

Clette, F., van der Linden, R., Cugnon, P., Berghmans, D., Foullon, C., Wauters, L., Verwichte, E., Hochedez, J.-F., and Vanlommel, P.: The Solar Influences Data Analysis Center: current status of expanding activities, in: Solar Variability: From Core to Outer Frontiers, ESA SP-506, 125-128, 2002.

Cliver, E. W., Kahler, S. W., and Reames, D. V.: Coronal Shocks and Solar Energetic Proton Events, Astrophys. J., 605, 902-910, 2004.

Crifo, F., Picat, J. P., and Cailloux, M.: Coronal transients - Loop or bubble, Sol. Phys., 83, 143-152, 1983.

Daly, E. J.: Space weather: a brief review, in: Solspa 2001, Proceedings of the Second Solar Cycle and Space Weather Euroconference, ESA SP-477, D17-D24, 2002.

Defise, J., Berghmans, D., Hochedez, J. F., Lecat, J. M., Mazy, E., Rochus, P. L., Thibert, T., Nicolosi, P., Pelizzo, M. G., Schuehle, U. H., Van der Linden, R. A. M., and Zhukov, A. N.: SWAP: Sun watcher using APS detector on-board PROBA-2, a new EUV off-axis telescope on a technology demonstration platform, in: Telescopes and Instrumentation for Solar Astrophysics, edited by: Fineschi, S. and Gummin, M. A., Proceedings of the SPIE, 5171, 143-154, 2004.

Delaboudiniere, J.-P., Artzner, G. E., Brunaud, J., Gabriel, A. H., Hochedez, J. F., Millier, F., Song, X. Y., Au, B., Dere, K. P., Howard, R. A., Kreplin, R., Michels, D. J., Moses, J. D., Defise, J. M., Jamar, C., Rochus, P., Chauvineau, J. P., Marioge, J. P., Catura, R. C., Lemen, J. R., Shing, L., Stern, R. A., Gurman, J. B., Neupert, W. M., Maucherat, A., Clette, F., Cugnon, P., and van Dessel, E. L.: EIT: Extreme-Ultraviolet Imaging Telescope for the SOHO Mission, Sol. Phys., 162, 291-312, 1995.

Domingo, V., Fleck, B., and Poland, A. I.: The SOHO Mission: an Overview, Sol. Phys., 162, 1-37, 1995.

Dryer, M.: Application of the classical initial-boundary value prob- lem to the Sun-to-Earth's space weather disturbances: some results and statistics, in: Solspa 2001, Proceedings of the Second Solar Cycle and Space Weather Euroconference, ESA SP-477, 9-6, 2002.

Dzifcáková, E., Kulinová, A., and Kosovichev, A. G.: A search for the relationship between flaring activity and subphotospheric flows, in: GONG+ 2002, Local and Global Helioseismology: the Present and Future, ESA SP-517, 263-266, 2003.

Emslie, A. G., Kucharek, H., Dennis, B. R., Gopalswamy, N., Holman, G. D., Share, G. H., Vourlidas, A., Forbes, T. G., Gallagher, P. T., Mason, G. M., Metcalf, T. R., Mewaldt, R. A., Murphy, R. J., Schwartz, R. A., and Zurbuchen, T. H.: Energy partition in two solar flare/CME events, J. Geophys. R., 109, A10 104, 2004.

Eyles, C. J., Simnett, G. M., Cooke, M. P., Jackson, B. V., Buffington, A., Hick, P. P., Waltham, N. R., King, J. M., Anderson, P. A., and Holladay, P. E.: The Solar Mass Ejection Imager (Smei), Sol. Phys., 217, 319-347, 2003.

Falconer, D. A.: A prospective method for predicting coronal mass ejections from vector magnetograms, J. Geophys. Res., 25185 25 190, 2001.

Feynman, J. and Gabriel, S. B.: On space weather consequences and predictions, J. Geophys. Res., 10 543-10 564, 2000.

Feynman, J. and Martin, S. F.: The initiation of coronal mass ejections by newly emerging magnetic flux, J. Geophys. Res., 100, 3355-3367, 1995.

Floyd, L., Newmark, J., Cook, J., Herring, L., and McMullin, D.: Solar EUV and UV spectral irradiances and solar indices, Journal of Atmospheric and Terrestrial Physics, 67, 3-15, 2005.

Fry, C. D., Sun, W., Deehr, C. S., Dryer, M., Smith, Z., Akasofu, S.I., Tokumaru, M., and Kojima, M.: Improvements to the HAF solar wind model for space weather predictions, J. Geophys. Res., 20 985-21 002, 2001.

Gaizauskas, V.: Preflare activity, Sol. Phys., 121, 135-152, 1989.

Gallagher, P. T., Moon, Y.-J., and Wang, H.: Active-Region Monitoring and Flare Forecasting - I. Data Processing and First Results, Sol. Phys., 209, 171-183, 2002.

Glover, A., Ranns, N. D. R., Harra, L. K., and Culhane, J. L.: The Onset and Association of CMEs with Sigmoidal Active Regions, Geophys. Res. Lett., 27, 2161, 2000.

GONG+ Team: Status of the GONG+ Camera Upgrade, in: SOHO9 Workshop on Helioseismic Diagnostics of Solar Convection and Activity, 1999.

Gopalswamy, N. and Kundu, M. R.: Estimation of the mass of a coronal mass ejection from radio observations, Astrophys. J. L., 390, L37-L39, 1992.

Gopalswamy, N., Lara, A., Yashiro, S., Kaiser, M. L., and Howard, R. A.: Predicting the 1-AU arrival times of coronal mass ejections, J. Geophys. Res., 29 207-29218, 2001a.

Gopalswamy, N., Yashiro, S., Kaiser, M. L., Howard, R. A., and Bougeret, J.-L.: Radio Signatures of Coronal Mass Ejection Interaction: Coronal Mass Ejection Cannibalism?, Astrophys. J. L., 548, L91-L94, 2001b.

Gosling, J. T.: Coronal mass ejections and magnetic flux ropes in interplanetary space, Washington D.C., AGU, Geophysical Monograph Series, 58, 343-364, 1990.

Gosling, J. T.: The solar flare myth, J. Geophys. Res., 98, $18937-$ $18950,1993$.

Gosling, J. T., Hildner, E., MacQueen, R. M., Munro, R. H., Poland, A. I., and Ross, C. L.: Mass ejections from the sun - A view from SKYLAB, J. Geophys. Res., 79, 4581-4587, 1974.

Gosling, J. T., Bame, S. J., McComas, D. J., and Phillips, J. L.: Coronal mass ejections and large geomagnetic storms, Geo- 
phys. Res. Lett., 17, 901-904, 1990.

Grechnev, V. V., Lesovoi, S. V., Smolkov, G. Y., Krissinel, B. B., Zandanov, V. G., Altyntsev, A. T., Kardapolova, N. N., Sergeev, R. Y., Uralov, A. M., Maksimov, V. P., and Lubyshev, B. I.: The Siberian Solar Radio Telescope: the current state of the instrument, observations, and data, Sol. Phys., 216, 239-272, 2003.

Haber, D. A., Hindman, B. W., and Toomre, J.: Interaction of solar subsurface flows with major active regions, in: ESA SP-517: GONG+ 2002, Local and Global Helioseismology: the Present and Future, 103-108, 2003.

Hale, G. E., Ellerman, F., Nicholson, S. B., and Joy, A. H.: The Magnetic Polarity of Sun-Spots, Astrophys. J., 49, 153-185, 1919.

Hill, F., Bolding, J., Toner, C., Corbard, T., Wampler, S., Goodrich, B., Goodrich, J., Eliason, P., and Hanna, K. D.: The GONG++ data processing pipeline, in: GONG+ 2002, Local and Global Helioseismology: the Present and Future, ESA SP-517, 295298, 2003.

Hochedez, J.-F., Jacques, L., Verwichte, E., Berghmans, D., Wauters, L., Clette, F., and Cugnon, P.: Multiscale activity observed by EIT/SoHO, in: Solspa 2001, Proceedings of the Second Solar Cycle and Space Weather Euroconference, ESA SP477, 115-118, 2002.

Hochedez, J.-F., Schmutz, W., Stockman, Y., Schühle, U., Ben Moussa, A., Koller, S., Haenen, K., Berghmans, D., Defise, J.-M., Halain, J.-P., Theissen, A., Delouille, V., Slemzin, V., Gillotay, D., Fussen, D., Dominique, M., Vanhellemont, F., McMullin, D., Kretzschmar, M., Mitrofanov, A., Nicula, B., Wauters, L., Roth, H., Rozanov, E., Rüedi, I., Wehrli, C., Soltani, A., Amano, H., Van der Linden, R., Zhukov, A., Clette, F., Koizumi, S., Mortet, V., Remes, Z., Petersen, R., Nesládek, M., D'Olieslaeger, M., Roggen, J., and Rochus, P.: LYRA: the Solar UV radiometer aboard the ESA Proba-2, Adv. Space Res., accepted, 2005.

Howard, R. A., Moses, J. D., and Socker, D. G.: Sun-Earth connection coronal and heliospheric investigation (SECCHI), in: Proc. SPIE 4139, Instrumentation for UV/EUV Astronomy and Solar Missions, edited by: Fineschi, S., Korendyke, C. M., Siegmund, O. H., and Woodgate, B. E., 259-283, 2000.

Hudson, H. S. and Cliver, E. W.: Observing coronal mass ejections without coronagraphs, J. Geophys. Res., 25 199-25 214, 2001.

Hundhausen, A. J., Sawyer, C. B., House, L., Illing, R. M. E., and Wagner, W. J.: Coronal mass ejections observed during the solar maximum mission - Latitude distribution and rate of occurrence, J. Geophys. Res., 89, 2639-2646, 1984.

Hundhausen, A. J., Holzer, T. E., and Low, B. C.: Do slow shocks precede some coronal mass ejections?, J. Geophys. Res., 92, 11 173-11 178, 1987.

Ippolito, A., Pommois, P., Zimbardo, G., and Veltri, P.: Magnetic connection from the Earth to the solar corona, flare positions and solar energetic particle observations., Astron. Astr., 438, 705711, 2005.

Jacobs, C., Poedts, S., Van der Holst, B., and Chané, E.: On the effect of the background wind on the evolution of interplanetary shock waves, Astron. Astr., 430, 1099-1107, 2005.

Jansen, F., Munakata, K., Duldig, M. L., and Hippler R.: MuSTAnG: Muon detectors - the real-time, groundbased forecast of geomagnetic storms in Europe, in: http://esa-spaceweather.net/spweather/workshops/./SPW_W3/ index.html, Space Weather Workshop: Looking Towards a Future European Space Weather Programme, 17-19 December 2001 ESTEC, Noordwijk, The Netherlands, 2001.
Jing, J., Yurchyshyn, V. B., Yang, G., Xu, Y., and Wang, H.: On the Relation between Filament Eruptions, Flares, and Coronal Mass Ejections, Astrophys. J., 614, 1054-1062, 2004.

Jones, H. P., Duvall, T. L., Harvey, J. W., Mahaffey, C. T., Schwitters, J. D., and Simmons, J. E.: The NASA/NSO spectromagnetograph, Sol. Phys., 139, 211-232, 1992.

Judge, D. L., McMullin, D. R., Gangopadhyay, P., Ogawa, H. S., Ipavich, F. M., Galvin, A. B., Möbius, E., Bochsler, P., Wurz, P., Hilchenbach, M., Grünwaldt, H., Hovestadt, D., Klecker, B., and Gliem, F.: Space weather observations using the SOHO CELIAS complement of instruments, J. Geophys. Res., 29 963-29968, 2001.

Kahler, S. W.: Solar flares and coronal mass ejections, Ann. R. Astron. Astr., 30, 113-141, 1992.

Kahler, S. W., Reames, D. V., and Sheeley, N. R.: Coronal Mass Ejections Associated with Impulsive Solar Energetic Particle Events, Astrophys. J., 562, 558-565, 2001.

Kaufmann, P., Giménez de Castro, C. G., Makhmutov, V. S., Raulin, J., Schwenn, R., Levato, H., and Rovira, M.: Launch of solar coronal mass ejections and submillimeter pulse bursts, J. Geophys. Res. S.-P., 108, 5-1, 2003.

Klein, L. W. and Burlaga, L. F.: Interplanetary magnetic clouds at 1 AU, J. Geophys. Res., 87, 613-624, 1982.

Klimchuk, J. A.: Proc. of the Chapman Conference on Space Weather, AGU Monograph series, edited by: Song, P., Siscoe, G., and Singer, H., Washington, D.C., AGU, 2000.

Krucker, S. and Lin, R. P.: Two Classes of Solar Proton Events Derived from Onset Time Analysis, Astrophys. J. L., 542, L61L64, doi:10.1086/312922, 2000.

Kunches, J.: Space weather activities at NOAA s Space Environment Center, in: COSPAR, Plenary Meeting, 2002.

Lean, J. L., Warren, H. P., Mariska, J. T., and Bishop, J.: A new model of solar EUV irradiance variability, 2. Comparisons with empirical models and observations and implications for space weather, J. Geophys. Res. S.-P., 108, 2-1, 2003.

Leerungnavarat, K., Ruffolo, D., and Bieber, J. W.: Loss Cone Precursors to Forbush Decreases and Advance Warning of Space Weather Effects, Astrophys. J., 593, 587-596, 2003.

Lemen, J. R., Duncan, D. W., Edwards, C. G., Friedlaender, F. M., Jurcevich, B. K., Morrison, M. D., Springer, L. A., Stern, R. A., Wuelser, J., Bruner, M. E., and Catura, R. C.: The solar X-ray imager for GOES, in: Telescopes and Instrumentation for Solar Astrophysics, edited by: Fineschi, S., Gummin, M. A., Proceedings of the SPIE, 5171, 65-76, 2004.

Lindsay, G. M., Luhmann, J. G., Russell, C. T., and Gosling, J. T.: Relationships between coronal mass ejection speeds from coronagraph images and interplanetary characteristics of associated interplanetary coronal mass ejections, J. Geophys. Res., 104, 12 515-12 524, 1999.

Luhmann, J. G., Solomon, S. C., Linker, J. A., Lyon, J. G., Mikic, Z., Odstrcil, D., Wang, W., and Wiltberger, M.: Coupled model simulation of a Sun-to-Earth space weather event, J. Atmos. S.P., 66, 1243-1256, 2004.

Marubashi, K.: Physics of Interplanetary Magnetic Flux Ropes: Toward Prediction of Geomagnetic Storms, Adv. Space Res., 26, 55-66, 2000.

McAllister, A. H., Martin, S. F., Crooker, N. U., Lepping, R. P., and Fitzenreiter, R. J.: A test of real-time prediction of magnetic cloud topology and geomagnetic storm occurrence from solar signatures, J. Geophys. Res., 29 185-29 194, 2001.

McIntosh, P. S.: The classification of sunspot groups, Sol. Phys., 125, 251-267, 1990. 
Moran, T., Gopalswamy, N., Dammasch, I. E., and Wilhelm, K.: A multi-wavelength study of solar coronal-hole regions showing radio enhancements, Astron. Astr., 378, 1037-1045, 2001.

Mouradian, Z., Soru-Escaut, I., and Pojoga, S.: On the two classes of filament-prominence disappearance and their relation to coronal mass ejections, Sol. Phys., 158, 269-281, 1995.

Nakajima, H., Nishio, M., Enome, S., Shibasaki, K., Takano, T., Hanaoka, Y., Torii, C., Sekiguchi, H., Bushimata, T., Kawashima, S., Shinohara, N., Irimajiri, Y., Koshiishi, H., Kosugi, T., Shiomi, Y., Sawa, M., and Kai, K.: New Nobeyama Radio Heliograph, Journal of Astrophysics and Astronomy Supplement, 16, 437, 1995.

Nitta, N. V., Lemen, J. R., and Hill, S. M.: Comparison of X-ray Images from Yohkoh/SXT and GOES-M/SXI, American Astronomical Society Meeting, Abstracts, 204, 2004.

Núñez, M., Fidalgo, R., Baena, M., and Morales, R.: The influence of active region information in the prediction of solar flares: an empirical model using data mining, Ann. Geophys., 23, 31293138, 2005.

Oberoi, D. and Kasper, J. C.: LOFAR: The potential for solar and space weather studies, Planet. Space Sci., 52, 1415-1421, 2004.

Pevtsov, A. A., Balasubramaniam, K. S., and Rogers, J. W.: Chirality of Chromospheric Filaments, Astrophys. J., 595, 500-505, 2003.

Quémerais, E. and Bertaux, J.: 14-day forecast of solar indices using interplanetary Lyman $\alpha$ background data, Geophys. Res. Lett., 29, 5-1, 2002.

Ramesh, R., Kathiravan, C., and Sastry, C. V.: Metric Radio Observations of the Evolution of a "Halo" Coronal Mass Ejection Close to the Sun, Astrophys. J. L., 591, L163-L166, 2003.

Reames, D. V.: Particle acceleration at the Sun and in the heliosphere, Space Sci. R., 90, 413-491, 1999.

Richardson, I. G., Cliver, E. W., and Cane, H. V.: Sources of geomagnetic storms for solar minimum and maximum conditions during 1972-2000, Geophys. Res. Lett., 28, 2569-2572, 2001.

Robbrecht, E. and Berghmans, D.: Automated recognition of coronal mass ejections (CMEs) in near-real-time data, Astron. Astr., 425, 1097-1106, 2004.

Robbrecht, E. and Berghmans, D.: Toward a holistic approach for CME monitoring, Proc. of the Chapman Conference on Solar Energetic Plasmas and Particles Turku 2004, Finland, AGU, Monograph series, accepted, 2005.

Rust, D. M.: Magnetic helicity, coronal mass ejections and the solar cycle, in: Solspa 2001, Proceedings of the Second Solar Cycle and Space Weather Euroconference, ESA SP-477, 39-41, 2002.

Sammis, I., Tang, F., and Zirin, H.: The Dependence of Large Flare Occurrence on the Magnetic Structure of Sunspots, Astrophys. J., 540, 583-587, 2000.

Scherrer, P. H. and SDO/HMI Team: The Helioseismic and Magnetic Imager for the Solar Dynamics Observatory, Bulletin of the American Astronomical Society, 34, 735, 2002.

Scherrer, P. H., Bogart, R. S., Bush, R. I., Hoeksema, J. T., Kosovichev, A. G., Schou, J., Rosenberg, W., Springer, L., Tarbell, T. D., Title, A., Wolfson, C. J., Zayer, I., and MDI Engineering Team: The Solar Oscillations Investigation - Michelson Doppler Imager, Sol. Phys., 162, 129-188, 1995.

Schrijver, C. J. and DeRosa, M. L.: Photospheric and heliospheric magnetic fields, Sol. Phys., 212, 165-200, 2003.

Schwadron, N. A. and McComas, D. J.: The Sheared Sub-Parker Spiral, AGU Fall Meeting, Abstracts, A4+, 2004.

Schwenn, R.: Mass ejections from the sun and their interplanetary counterparts, in: Solar Wind Conference, 45, 1995.
Schwenn, R., Dal Lago, A., Gonzalez, W. D., Huttunen, E., St.Cyr, C. O., and Plunkett, S. P.: A Tool For Improved Space Weather Predictions: The CME Expansion Speed, AGU Fall Meeting, Abstracts, A739+, 2001.

St. Cyr, O. C., Plunkett, S. P., Michels, D. J., Paswaters, S. E., Koomen, M. J., Simnett, G. M., Thompson, B. J., Gurman, J. B., Schwenn, R., Webb, D. F., Hildner, E., and Lamy, P. L.: Properties of coronal mass ejections: SOHO LASCO observations from January 1996 to June 1998, J. Geophys. Res., 18 169-18 186, 2000.

Steinegger, M., Denker, C., Goode, P. R., Marquette, W. H., Varsik, J., Wang, H., Otruba, W., Freislich, H., Hanslmeier, A., Luo, G., Chen, D., and Zhang, Q.: The new global high-resolution $\mathrm{H} \alpha$ network: preliminary results on the chromospheric differential rotation, in: SOHO 10/GONG 2000 Workshop: Helio- and Asteroseismology at the Dawn of the Millennium, ESA SP-464, 315-320, 2001.

Sterling, A. C. and Moore, R. L.: External and Internal Reconnection in Two Filament-Carrying Magnetic Cavity Solar Eruptions, Astrophys. J., 613, 1221-1232, doi:10.1086/423297, 2004.

Stone, E. C., Frandsen, A. M., Mewaldt, R. A., Christian, E. R., Margolies, D., Ormes, J. F., and Snow, F.: The Advanced Composition Explorer, Space Sci. Res., 86, 1-22, 1998.

Temerin, M. and Li, X.: A new model for the prediction of Dst on the basis of the solar wind, J. Geophys. Res., 107, 31-1, 2002.

Thornton, C. E. and Jones, H. P.: Comparison of Three Solar Magnetographs, Bulletin of the American Astronomical Society, 34, 1243, 2002.

Tobiska, W. K.: SOLAR2000 irradiances for climate change research, aeronomy and space system engineering, Adv. Space Res., 34, 1736-1746, 2004.

Toomre, J.: Overview - where do we stand with helioseismology?, in: ESA SP-517: GONG+ 2002. Local and Global Helioseismology: the Present and Future, 3-14, 2003.

Tsuneta, S., Acton, L., Bruner, M., Lemen, J., Brown, W., Caravalho, R., Catura, R., Freeland, S., Jurcevich, B., and Owens, J.: The soft X-ray telescope for the SOLAR-A mission, Sol. Phys., 136, 37-67, 1991.

Tsurutani, B. T., Gonzalez, W. D., Gonzalez, A. L. C., Tang, F., Arballo, J. K., and Okada, M.: Interplanetary origin of geomagnetic activity in the declining phase of the solar cycle, J. Geophys. Res., 100, 21 717-21 734, 1995.

Tylka, A. J., Boberg, P. R., Cohen, C. M. S., Dietrich, W. F., Maclennan, C. G., Mason, G. M., Ng, C. K., and Reames, D. V.: Flare- and Shock-accelerated Energetic Particles in the Solar Events of 2001 April 14 and 15, Astrophys. J. L., 581, L119L123, 2002.

Tylka, A. J., Cohen, C. M. S., Dietrich, W. F., Lee, M. A., Maclennan, C. G., Mewaldt, R. A., Ng, C. K., and Reames, D. V.: Shock Geometry, Seed Populations, and the Origin of Variable Elemental Composition at High Energies in Large Gradual Solar Particle Events, Astrophys. J., 625, 474-495, 2005.

Veronig, A. M., Temmer, M., and Hanslmeier, A.: The solar soft X-ray background flux and its relation to flare occurrence, Sol. Phys., 219, 125-133, 2004.

Wang, J., Shi, Z., Wang, H., and Lue, Y.: Flares and the Magnetic Nonpotentiality, Astrophys. J., 456, 861-878, 1996.

Wang, Y. M., Ye, P. Z., Wang, S., Zhou, G. P., and Wang, J. X.: A statistical study on the geoeffectiveness of Earth-directed coronal mass ejections from March 1997 to December 2000, J. Geophys. Res., 107, 2-1, doi:10.1029/2002JA009244, 2002.

Warren, H. P., Mariska, J. T., and Lean, J.: A new model of so- 
lar EUV irradiance variability: 1. Model formulation, J. Geophys. Res., 15 745-15 758, 2001.

Webb, D. F., Cliver, E. W., Crooker, N. U., Cry, O. C. S., and Thompson, B. J.: Relationship of halo coronal mass ejections, magnetic clouds, and magnetic storms, J. Geophys. Res., 74917508, 2000.

Weimer, D. R., Ober, D. M., Maynard, N. C., Burke, W. J., Collier, M. R., McComas, D. J., Ness, N. F., and Smith, C. W.: Variable time delays in the propagation of the interplanetary magnetic field, J. Geophys. Res., 107, 29-1, 2002.

Wen, Y. and Wang, J.: Low frequency radio signatures of coronal mass ejections, Progress in Astronomy, 22, 284-295, 2004.

Wheatland, M. S.: A Bayesian Approach to Solar Flare Prediction, Astrophys. J., 609, 1134-1139, 2004.

Wild, J. P., Smerd, S. F., and Weiss, A. A.: Solar Bursts, Ann. R. Astron. Astr., 1, 291-366, 1963.

Woods, T. N., Eparvier, F. G., Bailey, S. M., Chamberlin, P. C., Lean, J., Rottman, G. J., Solomon, S. C., Tobiska, W. K., and Woodraska, D. L.: Solar EUV Experiment (SEE): Mission overview and first results, J. Geophys. Res., 110(A01312), 2005.

Wuelser, J., Lemen, J. R., Tarbell, T. D., Wolfson, C. J., Cannon, J. C., Carpenter, B. A., Duncan, D. W., Gradwohl, G. S., Meyer, S. B., Moore, A. S., Navarro, R. L., Pearson, J. D., Rossi, G. R., Springer, L. A., Howard, R. A., Moses, J. D., Newmark, J. S., Delaboudiniere, J., Artzner, G. E., Auchere, F., Bougnet, M., Bouyries, P., Bridou, F., Clotaire, J., Colas, G., Delmotte, F., Jerome, A., Lamare, M., Mercier, R., Mullot, M., Ravet, M., Song, X., Bothmer, V., and Deutsch, W.: EUVI: the STEREOSECCHI extreme ultraviolet imager, in: Telescopes and Instrumentation for Solar Astrophysics, edited by: Fineschi, S. and Gummin, M. A., Proceedings of the SPIE, 5171, 111-122, 2004.
Yurchyshyn, V., Yashiro, S., Abramenko, V., Wang, H., and Gopalswamy, N.: Statistical Distributions of Speeds of Coronal Mass Ejections, Astrophys. J., 619, 599-603, 2005.

Yurchyshyn, V. B., Wang, H., Goode, P. R., and Deng, Y.: Orientation of the Magnetic Fields in Interplanetary Flux Ropes and Solar Filaments, Astrophys. J., 563, 381-388, 2001.

Zhang, J., Dere, K. P., Howard, R. A., and Bothmer, V.: Identification of Solar Sources of Major Geomagnetic Storms between 1996 and 2000, Astrophys. J., 582, 520-533, 2003.

Zhitnik, I. A., Bougaenko, O. I., Delaboudiniere, J.-P., Ignatiev, A. P., Korneev, V. V., Krutov, V. V., Kuzin, S. V., Lisin, D. V., Mitrofanov, A. V., Oparin, S. N., Oraevsky, V. N., Pertsov, A. A., Slemzin, V. A., Sobelman, I. I., Stepanov, A. I., and Schwarz, J.: SPIRIT X-ray telescope/spectroheliometer results, in: Solar Variability: From Core to Outer Frontiers, ESA SP-506, Proceedings, 915-918, 2002.

Zhukov, A. N.: Solar sources of geoeffective CMEs: a SOHO/EIT view, in: Coronal and Stellar Mass Ejections, edited by: Dere, K. P., Wang, J., and Yan, Y., Proceedings IAU Symposium 226, Cambridge University Press, 437-447, 2005.

Zhukov, A. N., Veselovsky, I. S., Clette, F., Hochedez, J.-F., Dmitriev, A. V., Romashets, E. P., Bothmer, V., and Cargill, P.: Solar Wind Disturbances and Their Sources in the EUV Solar Corona, in: Solar Wind Ten, AIP Conf. Proc. 679, 711-714, 2003.

Zuccarello, F.: Peculiar photospheric velocity fields and magnetic energy build-up, Astron. Astr., 257, 298-306, 1992. 